\title{
Solenoidal Magnetic Field Distribution based on Holzer Sensor
}

\author{
TONG Lei ${ }^{1}$, LI Chun-ran ${ }^{2}$ \\ 1.Department of College Foundation Education, Bohai University, Jinzhou , 121000, China \\ 2.School of Mathematics and Physics, Bohai University, Jinzhou, 121000, China
}

Email: 32229128@qq.com

\begin{abstract}
Keywords: Holzer Component, Magnetic Field, Magnetic Field Distribution, Experiment, Exciting Current
\end{abstract}

\begin{abstract}
The experimental measurement method for how to eliminate side effects of Holzer component has been mainly studied in this paper, Holzer sensor has been adopted to measure the relationship between exciting current and output Holzer voltage within electrified solenoid and the result that Holzer electric potential difference is in right proportion to magnetic induction intensity within the solenoid has been proven; important physical law of Holzer effect has been taken as standard value to calibrate or measure the sensitivity of Holzer sensor and get familiar with the characteristics and application of Holzer sensor; Holzer sensor has been adopted to measure the relationship between magnetic induction intensity within the electrified solenoid and axis position scale of solenoid.
\end{abstract}

\section{Introduction}

Postgraduate student Holzer from American the Johns Hopkins University has found out a magnetic phenomenon when studying the load bearing property of current carrying conductor in magnetic field in 1879, which is called as Holzer effect. Half a century later, people found out that semiconductor also has Holzer effect; moreover, the Holzer effect of semiconductor is stronger than metal. In recent 30 years, the Holzer sensor made of semiconductor material with high electron mobility rate has been widely applied to the research on magnetic field measurement and semiconductor material.

\section{Holzer effect}

Functions of Holzer component are as shown in picture 1 and picture 2. If current $I$ flows through the semiconductor wafer with thickness $d$ and magnetic field $B$ works on this semiconductor vertically, and then the electron flow direction will change with the function of Lorenz force, this phenomenon is called as Holzer effect. Electric potential difference produced between two horizontal planes $a, b$ of wafer and at the vertical direction of magnetic field $B$ is called as Holzer potential difference. 

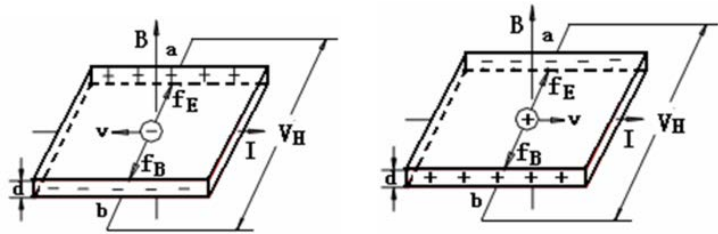

Figure 1, type N Holzer components Figure 2, type P Holzer components

Holzer potential difference is produced in this way: when current IH passes through Holzer component (assume it is $P$ model), the hole has certain drift velocity v; vertical magnetic field will produce a Lorentz force to motion charge.

$$
F_{B}=q \bullet(v \times B)
$$

In the formula, $q$ is electricity quantity of motion charge. Lorentz force produces lateral deflection to charge. Because the sample has boundary, so the deflected charge carrier will accumulate at the boundary and produce a lateral electric field $E$ until the force of electric field

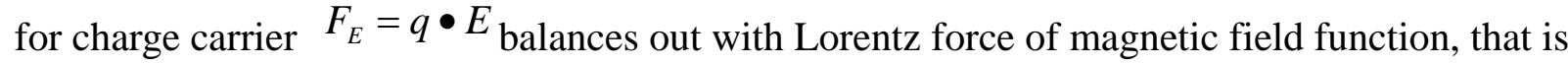

$$
q \bullet(v \times B)=q \bullet E
$$

At this moment, charge will not deflect when flows in sample. Holzer potential difference is established in the electric field.

If it is $N$ model sample, and then the lateral electric field is against the former, so the Holzer potential differences of $N$ model sample and $P$ model sample have different symbols. Based on this, conductive type of Holzer component can be judged.

Set the carrier concentration of $P$ type sample as $P$, width as $w$ and thickness as $d$. Through sample current $I_{S}=P \bullet q \bullet v \bullet w \bullet d$, introduce the velocity of hole $v=I_{S} / P \bullet q \bullet w \bullet d$ into formula (2) as:

$$
E=|v \times B|=\frac{I_{S} \bullet B}{p \bullet q \bullet w \bullet d}
$$

Multiply $w$ at two sides of the above formula to attain:

$$
V_{H}=E \bullet w=\frac{I_{S} \bullet B}{p \bullet q \bullet d}=R_{H} \bullet \frac{I_{S} \bullet B}{d}(4)
$$

In which, $R_{H}=\frac{1}{p \bullet q}$ is called as Holzer coefficient, which is written as the following in application

$$
V_{H}=K_{H} \bullet I_{S} \bullet B
$$




\section{$K_{H}=R_{H} / d=1 / p \bullet q \bullet d$ is called as sensitivity of Holzer component, the unit is $m V /(m A \bullet T)$}

And then the magnetic induction intensity is:

$$
B=\frac{V_{H}}{K_{H} \bullet I_{S}}(6)
$$

Generally, the bigger $K_{H}$, the better, $K_{H}$ and carrier concentration $P$ are inversely proportional. Carrier concentration within semiconductor is lower than metal carrier concentration, so all take semiconductor material as Holzer component. $K_{H}$ and material thickness $d$ are inversely proportional; therefore, all Holzer components are thin, at $0.2 \mathrm{~mm}$ generally (even at ten microns).

It can be seen from formula (5) that known the sensitivity of Holzer plate $K_{H}$, magnetic field $B$ can be calculated by measuring Holzer current $I_{S}$ and Holzer potential difference $V_{H}$ respectively, this is the principle of measuring magnetic field with Holzer effect.

Therefore, based on Holzer current $I_{S}$ and direction of magnetic field $B$, positive and negative Holzer voltage can be measured through experiment, and then confirm the positive or negative Holzer coefficient, that is to determine the positive and negative of carrier, which is an important method of studying semiconductor material. For the Holzer component of $N$ type semiconductor, conductive carrier is electron, both Holzer coefficient and sensitivity are negative; on the contrary, for Holzer component of $P$ type semiconductor, conductive carrier is hole, Holzer coefficient and sensitivity are positive.

\section{Side effects of Holzer component}

Generally, Holzer component has four lead wires, two of them are "current input terminals” of input Holzer component current, which are connected to adjustable power supply circuit; the other two are "Holzer voltage output terminals" of Holzer component, which are connected to digital voltage meter. It can be learnt from theory that when Holzer component is without magnetic field effect $(B=0), V_{H}=0$, but in real situation, the electric potential difference tested by digital voltage meter is not zero, which is called as residual voltage.

This is the potential difference caused by asymmetric electrode, uneven crystallization and thermo magnetic effect etc of semiconductor material. See details as following:

1) Unequal potential voltage drop $V_{0}$

Under the situation of current for Holzer component without magnetic field, electric potential difference should not exist between two voltage leading wires of Holzer theoretically. In fact, because the uneven surface of Holzer plate, differences exist in performance. Moreover, as two voltage leads of Holzer plat are not on the same equipotential surface, so one electric potential 
difference $V_{0}$ exists between two voltage leading wires as long as electrify Holzer plate even if there is no magnetic field. The direction of $V_{0}$ relates to the direction of current and irrelevant to the direction of magnetic field. $V_{0}$ is with the same order of magnitude as Holzer potential or even bigger. It ranks the top among all the additional potentials.

2) Etinghausen effect

After Holzer plate in magnetic field $B$ is electrified with current $I$, Lorentz forces borne by carrier are different as the transfer velocities of carrier are different. Therefore, orbital radiuses doing circular motion are different. The one with bigger velocity will move around bigger round track, while the one with smaller velocity will move around smaller track, which will cause one side of Holzer plate with more fast carriers and high temperature; the other side is with more slow carriers and low temperature. Temperature difference potential $V_{E}$ will appear due to the temperature difference between two sides. $V_{E}$ is in proportion to $I \bullet B$ product and the direction will change with $I$ and $B$ reversing.

3) Nernst

30 Nernst effect

Because the contact resistance of wire solder aroused by the current of Holzer component is different, after electrified with current $\mathrm{I}$, one end will absorb heat and the temperature will increase due to Peltier effect; the other end will release the heat and the temperature will decrease. Therefore, temperature difference appears. Uneven temperatures around the sample will cause temperature difference, and then cause additional potential $V_{N}$, whose direction relates to the direction of magnetic field and is irrelevant to the direction of current.

4) Righi-Leduc effect

The carrier transport rates of above thermal diffusion current are not the same. After placing Holzer component into magnetic field, temperature gradient will appear between voltage wires and then cause additional exponential $V_{R L}$. The direction of $V_{R L}$ is related to the direction of magnetic field and is irrelevant to the direction of current. In the practical application of Holzer component, the voltage compensation method in zero-magnetic field is usually adopted to remove the residual voltage of Holzer component.

During experimental measurement, to eliminate the influence of side effects, directions of $I_{S}$ and $B$ will be changed respectively. Record four groups of potential difference data (upward $K_{1}, K_{2}$ reversing switches are positive)

When $I_{S}$ is positive and $B$ is positive: $V_{1}=V_{H}+V_{0}+V_{E}+V_{N}+V_{R L}$

When $I_{S}$ is negative and $B$ is positive: $V_{2}=-V_{H}-V_{0}-V_{E}+V_{N}+V_{R L}$

When $I_{S}$ is negative and $B$ is negative: $V_{3}=V_{H}-V_{0}+V_{E}-V_{N}-V_{R L}$ 
When $I_{S}$ is positive and $B$ is negative: $V_{4}=-V_{H}+V_{0}-V_{E}-V_{N}-V_{R L}$

Calculate $V_{1}-V_{2}+V_{3}-V_{4}$ and take the average value, and then get $\frac{1}{4}\left(V_{1}-V_{2}+V_{3}-V_{4}\right)=V_{H}+V_{E}$

As $V_{E}$ and $V_{H}$ are with the same direction all the time, so it can't be eliminated by reversing method, but $V_{E}<<V_{H}$, so it can be neglected, and then

$$
V_{H}=\frac{1}{4}\left(V_{1}-V_{2}+V_{3}-V_{4}\right)
$$

It takes long time to establish temperature difference. Therefore, the measurement error can be decreased if adopts AC to make it unable to set up.

\section{Magnetic field distribution and magnetic induction intensity}

According to electromagnetic Biot-Savart law, magnetic induction intensity at central point of long straight solenoid axis is:

$$
\text { Bcenter }=\frac{\mu \bullet N \bullet I_{M}}{\sqrt{L^{2}+D^{2}}}
$$

Magnetic induction intensity at two ends of solenoid axis is:

$$
B a=\frac{1}{2} B_{b}=\frac{1}{2} \bullet \frac{\mu \bullet N \bullet I_{M}}{\sqrt{L^{2}+D^{2}}}
$$

In the formula, $\mu$ is magnetic permeability of magnetic medium. In vacuum, $\mu_{0}=4 \pi \times 10^{-7}(T \bullet m / A), N$ is total number of solenoids, $I_{M}$ is exciting current of solenoid, $L$ is length of solenoid and $D$ is the average diameter of solenoid.

\section{Conclusions}

Research on curent sensor system has been made based on above reasons with adoption of three different current sensor systems, including Holzer magnetic field sensor, anisotropic magnetoresistive field sensor and giant magnetostrictive material (GMM), in which the first two current sensor systems based on magnetic field sensor have developed independent sensor probe, data acquisition module, main control module, display module and host computer interface. Permalloy and copper foil have been adopted to make electromagnetic shielding for sensor probe, which can eliminate most of the external electromagnetic interference.

\section{Acknowledgements}

Study on the energy transfer and reaction mechanism of energetic materials at the early stage of initiation 2015020248. 


\section{Reference}

[1] Novickij J, Stankevič V, Balevičius S, et al. Manganite Sensor for Measurements of Magnetic Field Disturbances of Pulsed Actuators[J]. Solid State Phenomena, 2006, 113:459-464.

[2] Holzer E B. Simulation of the pion decay channel of a neutrino factory[J]. Nuclear Instruments \& Methods in Physics Research, 2002, 503(1-2):360-362.

[3] Hassanein A, Insepov Z, Norem J, et al. Effects of surface damage on rf cavity operation[J]. Office of Scientific \& Technical Information Technical Reports, 2006, 9(6):257-260.

[4] Myers F B, Lee L P. Innovations in optical microfluidic technologies for point-of-care diagnostics.[J]. Lab on A Chip, 2008, 8(12):2015-2031.

[5] Zhang Z, Li J, Fu L, et al. Magnetic molecularly imprinted microsensor for selective recognition and transport of fluorescent phycocyanin in seawater[J]. Journal of Materials Chemistry A Materials for Energy \& Sustainability, 2015, 3(14):7437-7444. 